stomach, lowering of frequency of development of erosions and ulcers of a stomach.

\section{AB0144 STUDYING OF THE EFFICIENCY OF THE SYNVISK IN OSTEOARTROSIS}

LG Groppa, MG Moshneaga. Department of Internal Medicine, State Medical and Pharmacy University, Kishinev, The Republic of Moldova, Kishinev, The Republic of Moldova

\subsection{6/annrheumdis-2001.406}

\section{Background}

Objectives At present for improvement of life quality in patients with osteoartrosis is necessary to solve some problems: precluding of development of degenerative process in cartilage of affected joint, relieving of pain and improvement of the function of the joint.

Methods For these aims we studied the efficiency of the chondroprotective drug Synvisk by Boenhringer Ingelheim in 25 patients with arthrosis of the knee in medium age of $61,2 \pm 1,7$ years, 16 women and 8 men, with duration of the disease 5,8 \pm 0,9 years.

Results Conform X-ray data the distribution of the patients by stages of the disease was as follow: I grade ? $20 \%$, II grade ? $64 \%$, and III grade ? $16 \%$. In experimental group the drug was administered intraarticular by blind method in dose of $2,0 \mathrm{ml}$ one time in a week, 3 injections for the course, which were repeated after 6 and 12 months. The control group was matched by gender, duration of disease and X-ray date; in this group was administrated placebo conforming same scheme.

Evaluation of the effectiveness and tolerability of compared drugs was carried out in evolution of clinical (pain, functional index by Lesquesne, volume of movement on affected joints), Xray parameters and functional methods (ultrasonography, scintigraphy with $\mathrm{Te} 99 \mathrm{~m}$.

In the basic group were mentioned decreasing of the pain intensity and improvement of the joint functions after single injection in one third of the patients, while in control groups similar cases were absent. After 3 courses of the treatment in the basic group the effects were obtained in $86,7 \%$ of the patients, including 33,3\% very good effect, in 53,4\% moderate effect and only in $13,3 \%$ the effects were absent. In control group there were no effect in $80 \%$ of the patients, and in $20 \%$ of the patients there were mentioned moderate effects; good effects were are not observed.

Conclusion Thus, a presented result suggests that administration of Synvisk lead to significant improvement of the life quality in the patients with arthrosis of the knee.

\section{AB0145 THE TREATMENT OF OSTEOARTHROSIS WITH DOLGIT CREAM IN KAZAKHSTAN}

BS Shakimova, WV Zhdanov, AA Zhankin. Rheumatology Unit, Kazakh State Medical University, Almaty, Kazakhstan

\subsection{6/annrheumdis-2001.407}

\section{Background}

\section{Objectives}

Methods The two weeks open trial of Dolgit (5\% ibuprofen) cream was carried out in 49 patients (pts) with osteoarthrosis (OA) (10 men and 39 women, age range 43-71 years) with disease history about 6,4 years. The 30 pts had gonarthrosis, 6 pts had OA of shoulder joint and 13 pts had lesion of interphalangeal joints. Patients were given an application of 5\% ibuprofen cream at the affected joints 3 times a day (600 mg of ibuprofen per day) during 2 weeks.

Results Results obtained after 1st cutaneous application showed that reducing of joint pain was marked in $40 \mathrm{~min}$ in 10 pts (77\%) with OA of interphalangeal joints and in an hour in half of pts with gonarthrosis and OA of shoulder joint. At $7^{\text {th }}$ day joint pain score reduced in all pts from 1,9 to 1,3 ( $p<0,05$ ), joint score - from 1,7 to 0,7 ( $\mathrm{p}=0,05)$, pain in movement had also reduced but not significantly $(\mathrm{p}>0,05)$.

At the end of trial joint pain score decreased to 0,5 ( $\mathrm{p}<$ $0,05)$ in pts with $\mathrm{OA}$ of interphalangeal joints and $\mathrm{OA}$ of shoulder joint, and in pts with gonarthrosis it reached 1 ( $\mathrm{p}>$ 0,05). Joint score had the similar trend.

Conclusion The final patient's treatment efficacy assessment was ?good? in 35 pts (71\%) and satisfactory in others. Tolerability of Dolgit cream was good, but in one case were signs of light skin irritation that disappeared without interruption of treatment.

Thus we consider the cutaneous application of Dolgit cream to be real alternative to systemic usage of NSAIDs in treatment of OA because of its good efficacy and tolerability.

\section{AB0146 OXIDATIVE STRESS EXPERIMENTS WITH SINOVITIS AND PERIOSTAL ACUPUNCTURE INFLUENCE}

AG Kurygin, AA Kratnov. Department of Therapy, Medical Academy, Yaroslavl, Russia

\subsection{6/annrheumdis-2001.408}

\section{Background}

Objectives For analysing different methods of influence to immunological mechanisms of inflammation with arthrosis complicated sinovitis various clinical and immunological tests with 105 patients have been analysed. For testing redox regulation of neutrophils and antioxidizing ferment system plasma [AFS] hemiluminescenciya of the blood [XL], XL of the blood plasma with $\mathrm{H} 2 \mathrm{O} 2$ [pXL], NBT-test have been used to define the contents of superoxiddismutase $[\mathrm{SOD}]$ in the neutrophils. The results have been analysed before and after the course of usual anti-inflammatory therapeutics and also together with periostal acupuncture.

Methods At ill with the various forms and the stages of arthrosis complicated sinovitis are presented a generality of changes oximetabolism neutrophils neutrophils and activity AFS plasma as the increase of its activity in compare with parameters of group of the control. The higher meanings oximetabolism neutrophils and SOD neutrophils are registered at ill female, and the activity AFS according to the data $\mathrm{pXL}$ of the blood plasma at men, that corresponds to distinction of clinical current of disease at different by weeding - "soft arthrosis to a condition" at men, that and painful arthrosis among women. The clinical distinction monoarthritis, arthrosis without knotes and knotes arthrosis at ill female proves to be true by distinction oximetabolism neutrophils, which consists in dynamics of growth of parameters of NBT test and XL of blood at the ill various forms. At the knotes arthrosis, as against other forms arthrosis complicated sinovitis, increase of parameters of the NBT-test is observed at backlog of growth XL of blood, probably connected with blockade receptors neutrophils by immune complexes, the quantity of which is increased in blood regular with growth of pain of the form arthrosis complicated sinovitis. At ill knotes arthrosis by the form of disease arises infrigment of antioxidizing ferment 
system, connected with absence of compensatory growth of activity neutrophils SOD and AFS plasma at increase of oximetabolism in neutrophils, observable at other forms arthrosis. Infrigment of AFS plasma and decrease microbicidal activity neutrophils at ill female with knotes arthrosis complicated sinovitis is supposed to be connected to violation in regulation lymphocytes and granulocytes. At the study of morphological structure of blood ill arthrosis increase of relative quantity lymphocytes and decrease neutrophils was found out at the knotes arthrosis at ill female, that is an attribute of painful process.

The given infrigment of morphological structure of blood at ill female with knotes arthrosis complicated sinovitis correlates with growth redox activity, that is possibly connected to induction aggregation neutrophils under influence of immune complexes. Violation oximetabolism neutrophils at ill female with knotes arthrosis complicated sinovitis is the most expressed in the age of 41-50 years old is probably connected with infrigment of gonad system at the women in period, previous menopause and corresponds to data of the literature on high activity arthrosis in this age. The changes occuring on influence laserotherapy are connected to amplification of activity AFS plasma, that occurs at photochemical reactivate by radiation laser ferum and copper contain ferments, at insignificant influence on oximetabolism neutrophils.

Results The greatest positive dynamics redox regulation of neutrophils and activity antioxidizing ferment systems at ill arthrosis occured at acupuncture treatment, as monotherapy, also in combination with diclofenac. Given changes were combined with normalisation of morphological structure of blood. Thus the periostal acupuncture effect is connected not only with the releasing of pain but also with immunomodulation mechanism normalising the neutrophils activity.

Conclusion

\section{AB0147 DRUG TREATMENT OF OSTEOARTHRITIS: ASPECTS OF SYMPTOM AND STRUCTURE MODIFICATION}

KK Förster, KW Schmid. Department of Clinical Research, Opfermann Arzneimittel GmbH, Wiehl, Germany

\subsection{6/annrheumdis-2001.409}

Background "Osteoarthritis (OA) is a degenerative disease of the joint cartilage. It is of diverse aetiology and obscure pathogenesis. Clinically, OA is characterised by joint pain, tenderness, movement limitation, occasional effusion, and variable degrees of local inflammation, but without systemic effects. Therapeutically, OA is characterised by a lack of specific healing". ${ }^{1}$ Therefore, treatment of $\mathrm{OA}$ is mainly a symptomatic one, a specific treatment, with a demonstrable causal effect on the pathological process, is still on the way to be proven clinically.

Objectives Historically, OA has been viewed as a disease for which little can be done to block its progression. However, the significant increase in understanding the underlying pathologic processes has substantially increased the number of potential targets for drug intervention (Howell et al. 1995). In order to clearly define OA therapies, recently it was agreed to classify OA drugs as follows:

- Symptom modifying drugs (with no detectable effect on the structural changes of the disease).

- Structure modifying drugs (which interfere with the progression of $\mathrm{OA}$ ).
Methods Based on published placebo as well as reference controlled clinical studies, evidence is assessed for the classification of drugs used in the treatment of osteoarthritis.

Results Besides corticosteroids, classical NSAIDs, and analgesics, symptom modifying OA pharmacotherapy includes Ademethionine, ASU, Chondroitin sulfate (CS), Diacerein, Glucosamine sulfate (GS, dona 2oo-S), Hyaluronic acid (HA), and Oxaceprol. All show an anti-inflammatory effect, but unlike classical NSAIDs most of them do not inhibit lip- or cyclo-oxygenase. Therefore, they have been described as nonclassical NSAIDs. ${ }^{2}$

On the other hand, experience based on in vitro studies and animal models shows that some drugs used in the treatment of OA, rather than having a beneficial symptomatic effect, may accelerate or exacerbate the pathological changes of OA (Brandt et al. 1998). This potential drug induced cartilage change for the worse has stimulated interest in pharmacologic agents which may positively influence the pathogenetic mechanisms in OA. In case these agents would be disease modifying (Lequesne et al. 1994) or structure modifying drugs (Dougados et al. 1996). Many substances have already been tested experimentally. However, a specific OA treatment has very rarely been confirmed so far in hypothesis testing clinical studies.

Concerning potential structure modification of CS and HA, there are only a few pilot clinical studies. Concerning GS, it was proven recently that during long-term treatment the drug exerts combined structure-modifying and symptom-modifying effects so that it is to suggest that GS could be a disease modifying agent in osteoarthritis (Reginster et al. 2001).

Conclusion A future challenge will be the design and implementation of further appropriate clinical trials (Dieppe 1994), with longer follow-up and with different designs, to assess whether disease modification leads to reduced need of joint surgery and prolonged time to substantial disability, respectively (Reginster et al. 1996).

\section{REFERENCES}

1 Mankin HJ, Brandt KD, Shulman LE. Workshop on etiopathogenesis of osteoarthritis. Proceedings and recommendations. J Rheumatol. 1986;13:1127-60

2 Förster KK. Drug treatment of osteoarthritis: clinical aspects. In: Grifka J, OgilvieHarris DJ, eds. Osteoarthritis. Berlin Heidelberg New York: Springer-Verlag, 2000: 66-81

3 Reginster JY, Deroisy R, Paul I, Lee RL, Henrotin Y, Giacovelli G, Dacre J, Rovati LC, Gosset C. Long-term effects of glucosamine sulphate on osteoarthritis progression: a randomised, placebo-controlled clinical trial. Lancet 1999;i:251-6

\section{AB0148 CHIROPRACTIC MANAGEMENT OF SHOULDER CAPSULITIS AND KNEE OSTEOARTHRITIS}

AP Rozin. The B.Shine Department of Rheumatology, Rambam Medical Center, Haifa, Israel

10.1136/annrheumdis-2001.410

Background The treatment of osteoarthritis (OA) of the knees and adhesive capsulitis of the shoulders (SAC) is often disappointing. Recent EULAR clinical guidlines emphasise the role of non-pharmacological treatment modalities for knee OA. ${ }^{1}$ Since 1990 some reports confirmed the benefit of chiropractic management for low back pain., ${ }^{2,3}$ The theory of the vertebrone laid by Gutzeit (1951) related vertebrogenic nerve involvement with target tissue pathology. Chiropractic method of Kasyan was devised and originally modified for the treatment of spine, shoulders and degenerative disease of the knees. ${ }^{4,5}$

Objectives To assess the results of modified Kasyan chiropractic treatment for osteoarthritis of the knee and for adhesive capsulitis of the shoulder. 